Cite this: Chem. Commun., 2014

50,833

Received 29th July 2013,

Accepted 9th November 2013

DOI: $10.1039 / c 3 c c 45791 e$

www.rsc.org/chemcomm

\section{Charge transfer-assisted self-limited decyanation reaction of TCNQ-type electron acceptors on $\mathrm{Cu}(100) \dagger$}

\author{
Christian Urban, ${ }^{a}$ Yang Wang, ${ }^{\mathrm{bc}}$ Jonathan Rodríguez-Fernández, ${ }^{\mathrm{a}}$ Raúl García, \\ M. Ángeles Herranz, ${ }^{d}$ Manuel Alcamí, ${ }^{c}$ Nazario Martín, ${ }^{\text {bd }}$ Fernando Martín, ${ }^{\text {bc }}$ \\ José M. Gallego, ${ }^{\text {be }}$ Rodolfo Miranda ${ }^{a b}$ and Roberto Otero*ab
}

TCNQ derivatives adsorbed on a metal surface undergo a selflimited decyanation reaction that only affects two out of the four cyano groups in the molecule. Combined Scanning Tunneling Microscopy/X-ray Photoelectron Spectroscopy experiments and Density Functional Theory calculations relate the self-limiting behavior to the transfer of electrons from the metal to the molecule.

The development of surface chemistry has been historically linked with the need to understand and improve heterogeneous catalysts for industrial applications. ${ }^{1}$ In a few selected cases, such investigations have resulted in the first-principles design of new and improved catalysts through a profound understanding of the role that solid surfaces play in determining the adsorption geometry, charge state and reaction pathways of the reactant molecules. ${ }^{2}$ However, most of the successful stories so far have involved only relatively small and uncomplicated molecules, such as carbon monoxide, methane or ammonia, ${ }^{2}$ although significant progress is also being made in more complex molecules. ${ }^{3}$

In recent years, the interest in the catalytic role of solid surfaces in the reaction of more complex organic molecules has been renewed, due to the possibility of finding new synthetic routes towards 1 and 2D well-ordered polymers. ${ }^{4}$ However, the atomistic details of how the atoms at the surface change the reactivity of significantly more complex organic adsorbates involved in such reactions are still not fully understood. ${ }^{5}$ In most of the reactions previously described in the literature, the organic adsorbates are provided with specific functional groups which react at a given temperature all at once, allowing for the formation of networks with

\footnotetext{
${ }^{a}$ Dep. de Física de la Materia Condensada, Universidad Autónoma de Madrid, Madrid, Spain. E-mail: roberto.otero@uam.es; Fax: +34 91497 3961; Tel: +34 914976462

${ }^{b}$ Instituto Madrileño de Estudios Avanzados en Nanociencia (IMDEA-Nano), Madrid, Spain

${ }^{c}$ Dep. de Quimica, Universidad Autónoma de Madrid, Madrid, Spain

${ }^{d}$ Dep. de Química Orgámica, Universidad Complutense de Madrid, Madrid, Spain

${ }^{e}$ Instituto de Ciencia de Materiales de Madrid (ICMM-CSIC), Madrid, Spain

$\dagger$ Electronic supplementary information (ESI) available: Experimental and computational details. See DOI: 10.1039/c3ec45791e
}

specific geometries. In some cases, however, it might be useful to preserve some of these functional groups, for example, to provide the resulting species with electron donor or acceptor characteristics.

In this communication we report on the thermally-induced self-limited decyanation of the organic electron acceptor 15,15,16,16-tetracyano-6,13-pentacene-p-quinodimethane (TCPQ) when adsorbed on the $\mathrm{Cu}(100)$ surface, studied by an in situ combination of Variable-Temperature Scanning Tunneling Microscopy (VT-STM) and X-ray Photoelectron Spectroscopy (XPS) together with Density Functional Theory (DFT) calculations. We show that, upon adsorption, charge-transfer from the metal substrate to the organic adsorbate controls the molecular conformation, leading to an adsorption geometry in which only two cyano groups are bonded to the surface (hereinafter referred to as lower cyano groups), while the other two point away from it (upper cyano groups). We will demonstrate that the breaking of the symmetry among the upper and lower cyano groups is so severe that two cyano groups can be selectively eliminated from each molecule, limiting any further progress in the reaction. These results cannot be properly understood unless the role of the surface as a source of electrons is properly accounted for. Notice that related charge-transfer processes have been found to play a very important role in the way by which molecules of the TCNQ family adsorb on metallic surfaces and in the way by which they interact with each other on such surfaces. ${ }^{6}$

Fig. 1a shows the gas-phase conformation of the TCPQ molecules in the neutral state. Due to steric repulsion between the cyano groups and the $\mathrm{H}$ atoms in the peripheral positions, these molecules are not planar in the gas phase, but adopt a butterfly-shaped structure with the dicyanomethylene groups and the aromatic rings folded in opposite directions. ${ }^{7,8}$ Upon deposition on $\mathrm{Cu}(100)$ at room temperature (RT), the molecules are not observed to aggregate into ordered islands as TCNQ does, ${ }^{6}$ but they stay as isolated entities (see Fig. 1a). The molecules display a crossshape appearance, with two bright lobes at both sides of a darker cigar-shaped structure. Comparison of the images with those corresponding to the related 13,13,14,14-tetracyano-5,12-tetracenep-quinodimethane (TCTQ, Fig. 1b) molecules demonstrates that 

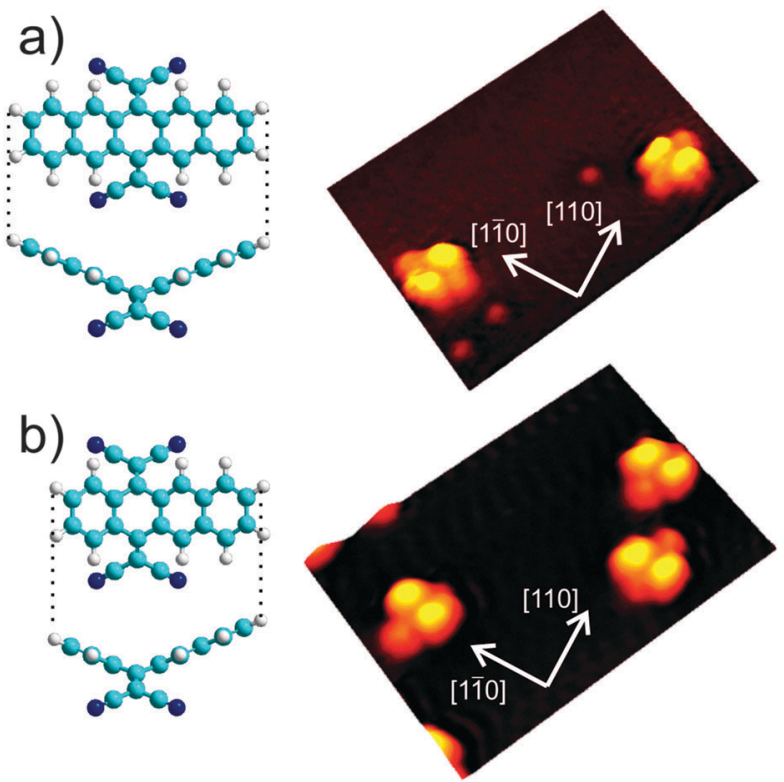

Fig. 1 STM $\left(6.3 \times 4.4 \mathrm{~nm}^{2}\right)$ images of isolated molecules of (a) TCPQ and (b) TCTQ on the $\mathrm{Cu}(100)$ surface. (a) $V_{\mathrm{b}}=-0.83 \mathrm{~V}, I=0.64 \mathrm{nA}$; (b) $V_{\mathrm{b}}=$ $-1.79 \mathrm{~V}, I=0.24 \mathrm{nA}$.

the dicyanomethylene groups correspond to the bright lobes, while the cigar shaped structure must arise from the aromatic rings.

State-of-the-art van der Waals-corrected DFT calculations performed with the smallest member of the molecular family, 11,11,12,12-tetracyano-9,10-anthra- $p$-quinodimethane (TCAQ), ${ }^{9}$ show that the minimum energy configuration is such that only two cyano groups are bonded to the substrate, while the other two point out of the surface plane (Fig. 2a). STM images of such adsorption geometry, calculated following the Tersoff-Hamann approximation, reveal the upper cyano groups as bright protrusions, while the planarized polyarene moiety appears as a darker cigar structure (Fig. 2b), in agreement with the experimental results described above.

Inspection of the molecular conformation obtained from the DFT calculations show that, similarly to the case of TCNQ on $\mathrm{Cu}(100),{ }^{6}$ the adsorbed molecule is severely distorted when compared with the gas phase conformation of Fig. 1. In this case the steric hindrance between the polyarene moiety and the dicyanomethylene ends is released by rotating the whole dicyanomethylene ends around the B3 bond (Fig. 2c). Such rotation would be energetically expensive for the molecule in the neutral state, suggesting that TCPQ, much like in the TCNQ case, uptakes electronic charge from the metal surface.

The previous assertion is confirmed by a Bader analysis of our DFT results, which shows that TCAQ takes about $1.99 \mathrm{e}^{-}$from the surface. XPS measurements also corroborate the transfer of charge from the metal surface to the molecule (see Fig. 2d). While for submonolayer coverages the $\mathrm{N}$ signal shows a single maximum at $398.0 \mathrm{eV}$, for a coverage $\theta=1.2 \mathrm{ML}$ a second component at higher binding energy ( $399.4 \mathrm{eV}$ ) is clearly noticeable. The intensity of this component increases with coverage, and it is the one that dominates the spectrum for $\theta=2.6 \mathrm{ML}$. A similar shift of $\sim 0.9-1.4 \mathrm{eV}$ towards lower binding energies for submonolayer coverages was
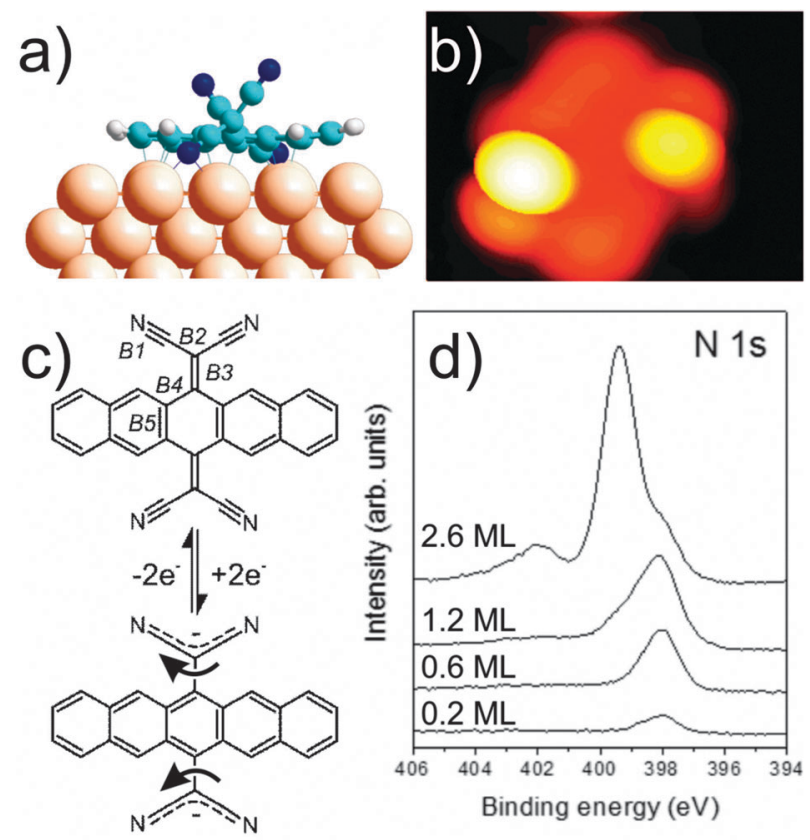

Fig. 2 (a) Side view of the relaxed conformation of TCAQ on $\mathrm{Cu}(100)$ showing that the dicyanomethylene end groups are rotated around bond B3, consistent with charge-transfer from the metallic substrate. (b) The simulated STM image based on the adsorption geometry shown in (a). (c) Modification in the bond conjugation of TCPQ upon the uptake of $2 \mathrm{e}^{-}$, and the concomitant change in conformational freedom. (d) X-ray photoelectron spectra $(h \nu=1486 \mathrm{eV})$ of the $\mathrm{N} 1 s$ core level of TCPQ/Cu(100) at different coverages. TCPQ molecules in direct contact with the substrate show a core level shift towards lower binding energies, in good agreement with the electron acceptor character of TCPQ.

also observed after depositing TCNQ on $\mathrm{Ni}(111)^{10,11}$ or $\mathrm{Cu}(100){ }^{6}$ and was attributed to charge transfer from the substrate to the molecule. The width of the peak for submonolayer coverage is also significantly larger than for the multilayer component $(1.36 \mathrm{eV}$ and $1.16 \mathrm{eV}$, respectively), which can be explained by the asymmetric situation of the upper and lower cyano groups at submonolayer coverage.

The breaking of the symmetry between the four cyano groups raises expectations of different behavior in subsequent reactions for the adsorbed TCPQ molecules. We have performed a series of experiments in which the surface is annealed to increasing temperatures up to $500 \mathrm{~K}$, and subsequently cooled down for STM and XPS examination. For annealing temperatures higher than $380 \mathrm{~K}$ we observe that the pentacene moiety is preserved in all the molecular units, but an increasing number of molecules with one (Fig. 3a, blue arrow) or two (Fig. 3a, green arrow) missing bright lobes can be found as the annealing temperature increases. A statistical analysis of the number of missing lobes as a function of temperature extracted from large scale images (see Fig. S1, ESI $\dagger$ ) can be seen in Fig. 3e. This evolution is consistent with the expected one for a barrier height of about $1.1 \mathrm{eV}$ (assuming reasonable prefactors, see Table S1, ESI $\dagger$ ). At $490 \mathrm{~K}$ all the molecules observed in the STM images have lost the two upper cyano groups. Comparison between the XPS spectra of C1s and N1s core levels before and after annealing to $490 \mathrm{~K}$ reveals that the vanishing of the bright lobes is associated with the loss of $50 \%$ of the initial nitrogen and about 

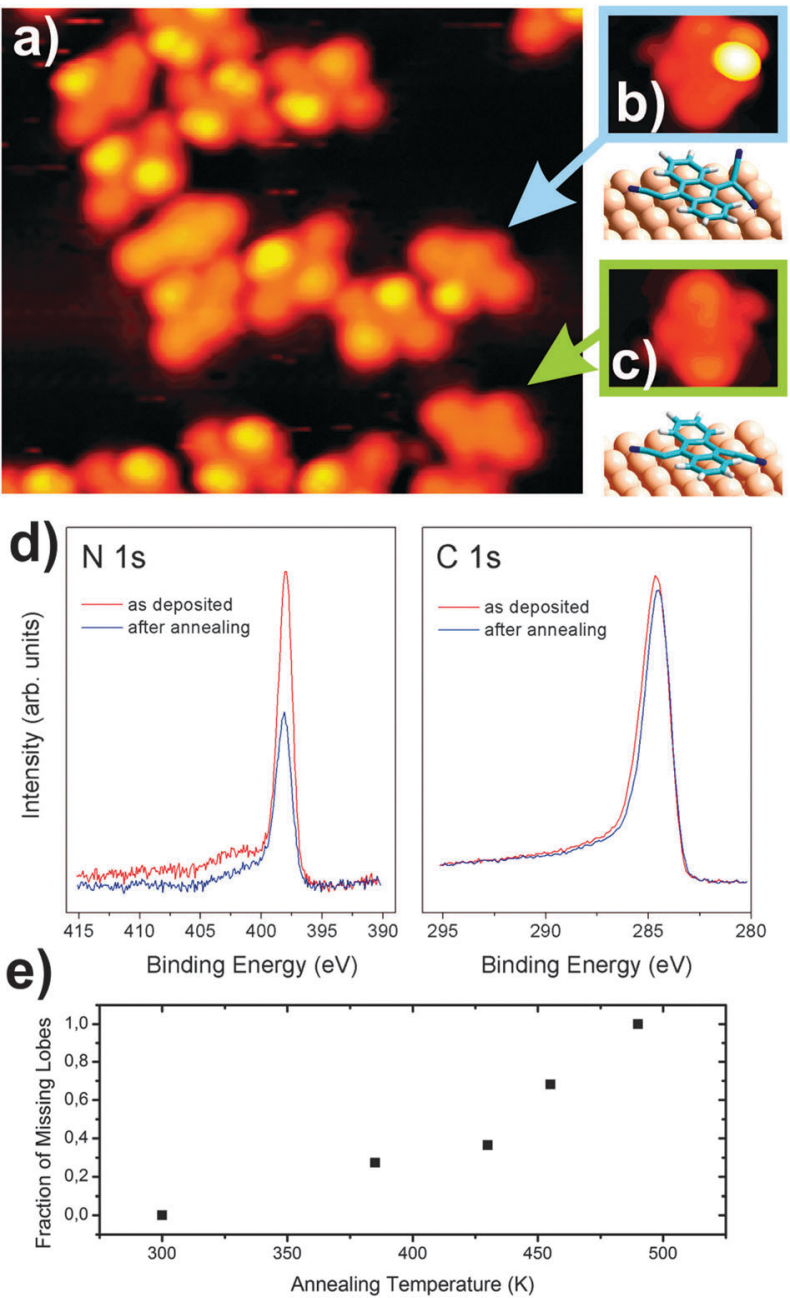

Fig. 3 (a) STM image $\left(9.5 \times 6.8 \mathrm{~nm}^{2}\right)$ of the $\mathrm{Cu}(100)$ surface after annealing to $380 \mathrm{~K} \sim 0.5 \mathrm{ML}$ of TCPQ deposited at room temperature. Molecules can now be found which have lost either one (blue arrow) or two (green arrow) bright lobes. (b, c) Calculated relaxed geometries for the surface-stabilized radicals resulting from the elimination of one (b) or two (c) cyano groups from the TCAQ molecule, and the corresponding calculated STM images. (d) X-Ray photoemission spectra ( $h \nu=1468 \mathrm{eV})$ of the N1s (left) and C1s (right) core levels before (red line) and after (blue line) the annealing to $490 \mathrm{~K}$. (e) Statistical analysis of the number of missing lobes as a function of the annealing temperature.

$10 \%$ of the initial carbon (see Fig. 3d). This is consistent with the molecules losing two cyano groups per molecule ( 2 carbon and 2 nitrogen atoms out of 28 and 4 carbon and nitrogen atoms in the intact molecules respectively). Notice that losing entire dicyanomethylene groups is incompatible with our experimental results, since no nitrogen would be left on the surface upon total disappearance of the bright lobes. The decyanation reaction is also supported by comparison of the experimental images (Fig. 3a) with simulated STM images arising from DFT calculations for singly (Fig. 3b) and doubly (Fig. 3c) decyanated TCAQ radicals adsorbed on $\mathrm{Cu}(100)$.

We have thus demonstrated that TCPQ molecules adsorbed on $\mathrm{Cu}(100)$ undergo decyanation of the dicyanomethylene ends. Notice that only two cyano groups have been lost during the process, while the other two cyano groups remain unperturbed. This is thus an example of a self-limited reaction: the decyanation reaction proceeds no further, even though the four cyano groups seem to be equivalent from the molecular symmetry. Of course, the reason for such behavior is the interaction between the cyano groups and the surface, which is very strong for the lower cyano groups, but very weak for the upper ones. Thus, while the energy barrier for the upper cyano groups to detach from the molecular backbone can be expected to amount to the energy cost for breaking the bond B2 (see Fig. 2c), a similar process in the lower cyano groups must also pay the energy price to separate the cyano groups from the surface. Charge-transfer is thus essential in determining the reactivity of the adsorbed organic species. Finally, we would like to point out that understanding the new reactions that appear to occur only at solid surfaces might actually lead to new synthetic pathways that could find their way towards applications in the near future.

We thank the CCC-UAM and the RES for allocation of computer time. Our work has been supported by the MINECO of Spain (MAT2009-13488, FIS2010-18847, FIS2010-15127, FIS2012-33011, CTQ2010-17006, CTQ2011-24652/BTQ), Comunidad de Madrid (Nanobiomagnet S2009/MAT-1726, Madrisolar-2 S2009/PPQ-1533), CONSOLIDER-INGENIO on Molecular Nanoscience (CSD200700010) and European Union (SMALL PITN-GA-2009-23884).

\section{Notes and references}

1 G. Somorjai, Introduction to Surface Chemistry and Catalysis, Wiley, Hoboken, 2nd edn, 2010; G. Ertl, Reactions at Solid Surfaces, Wiley, Hoboken, 2009.

2 F. Besenbacher, I. Chorkendorff, B. S. Clausen, B. Hammer, A. M. Molenbroek, J. K. Nørskov and I. Stensgaard, Science, 1998, 279, 1913; G. Ertl, D. Prigge, R. Schloegl and M. Weiss, J. Catal., 1983, 79, 359; B. K. Min and C. M. Friend, Chem. Rev., 2007, 107, 2709.

3 Z. Ma and F. Zaera, Surf. Sci. Rep., 2006, 61, 229.

4 L. Grill, M. Dyer, L. Lafferentz, M. Persson, M. V. Peters and S. Hecht, Nat. Nanotechnol., 2007, 2, 687; S. Weigelt, C. Busse, C. Bombis, M. M. Knudsen, K. V. Gothelf, E. Lægsgaard, F. Besenbacher and T. R. Linderoth, Angew. Chem., Int. Ed., 2008, 47, 4406; N. A. A. Zwaneveld, R. Pawlak, M. Abel, D. Catalin, D. Gigmes, D. Bertin and L. Porte, J. Am. Chem. Soc., 2008, 130, 6678; J. Cai, P. Ruffieux, R. Jaafar, M. Bieri, T. Braun, S. Blankenburg, M. Muoth, A. P. Seitsonen, M. Saleh, X. Feng, K. Müllen and R. Fasel, Nature, 2010, 466, 470; S. Boz, M. Stöhr, U. Soydaner and M. Mayor, Angew. Chem., Int. Ed., 2009, 48, 3179; J. A. Lipton-Duffin, J. A. Miwa, M. Kondratenko, F. Cicoira, B. G. Sumpter, V. Meunier, D. F. Perepichka and F. Rosei, Proc. Natl. Acad. Sci. U. S. A., 2010, 107, 11200; M. Matena, T. Riehm, M. Stöhr, T. A. Jung and L. H. Gade, Angew. Chem., Int. Ed., 2008, 47, 2414.

5 M. Bieri, M. Nguyen, O. Gröning, J. Cai, M. Treier, K. Aït-Mansour, P. Ruffieux, C. A. Pignedoli, D. Passerone, M. Kastler, K. Müllen and R. Fasel, J. Am. Chem. Soc., 2010, 132, 16669; M. Int'l Veld, P. Iavicoli, S. Haq, D. B. Amabilino and R. Raval, Chem. Commun., 2008, 1536; D. Zhong, J. Franke, S. K. Podiyanachari, T. Blömker, H. Zhang, G. Kehr, G. Erker, H. Fuchs and L. Chi, Science, 2011, 334, 213.

6 T. Tseng, C. Urban, Y. Wang, R. Otero, S. L. Tait, M. Alcamí, D. Écija, M. Trelka, J. M. Gallego, N. Lin, M. Konuma, U. Starke, A. Nefedov, A. Langner, C. Wöll, M. Á. Herranz, F. Martín, N. Martín, K. Kern and R. Miranda, Nat. Chem., 2010, 2, 374.

7 R. Viruela, P. M. Viruela, E. Orti and N. Martín, Synth. Met., 1995, 70, 1031.

8 N. Martin, R. Behnisch and M. Hanack, J. Org. Chem., 1989, 54, 2563; N. Martin and M. Hanack, J. Chem. Soc., Chem. Commun., 1988, 1522.

9 E. Ortí, R. Viruela and P. M. Viruela, J. Phys. Chem., 1996, 100, 6138. 10 J. Giergiel, S. Wells, T. A. Land and J. C. Hemminger, Surf. Sci., 1991, $255,31$.

11 J. M. Lindquist and J. C. Hemminger, Chem. Mater., 1989, 1, 72; J. M. Lindquist and J. C. Hemminger, J. Phys. Chem., 1988, 92, 1394. 